\title{
Spatial Long-Term Memory Is Related to Mossy Fiber Synaptogenesis
}

\author{
Victor Ramírez-Amaya, Israela Balderas, Jimena Sandoval, Martha L. Escobar, and \\ Federico Bermúdez-Rattoni \\ Departamento de Neurociencias, Instituto de Fisiología Celular, Universidad Nacional Autónoma de México, \\ 04510 México
}

Structural synaptic changes have been suggested to underlie long-term memory formation. In this work, we investigate if hippocampal mossy fiber synaptogenesis induced by water maze overtraining can be related with long-term spatial memory performance. Rats were trained in a Morris water maze for one to five identical daily sessions and tested for memory retrieval 1 week and 1 month after training. After the last test session, the rat brains were obtained and processed for Timm's staining to analyze mossy fiber projection. The behavioral results showed that with more training, animals showed a better performance in the memory tests, and this performance positively correlates with Timm's staining in the stratum oriens. Furthermore, with the use of the NMDA antagonist MK801 before, but not after acquisition, water maze spatial memory was impaired. Increased Timm's staining in the stratum oriens was observed in the animals treated with MK801 after acquisition but not in those treated before. Finally, we observed that mossy fiber synaptogenesis occurs mainly in the septal region of the dorsal hippocampus, supporting the idea that this anterior region is important for spatial memory. Altogether, these results suggest that mossy fiber synaptogenesis can be related with spatial long-term memory formation.

Key words: mossy fiber sprouting; structural changes; hippocampus; long-term memory; dizocilpine; synaptogenesis; CA3; spatial learning
Learning and memory result from changes in the neural representation of stimuli (Dudai, 1989) through plastic events that modify the way neurons communicate with each other (Bear, 1996). Plastic events can include changes in the structure, distribution and number of synapses, and it has been suggested that these morphological changes underlie memory formation (Rusakov et al., 1997; Woolf, 1998; Klintsova and Greenough, 1999).

The hippocampus plays a crucial role in the performance of spatial tasks, and the activity of its cells has been suggested to be related with spatial representation (Nadel and Eichenbaum, 1999). This structure is particularly plastic, where morphological changes such as synaptogenesis and neurogenesis occur in the adult brain (Ben-Ari and Represa 1990; Derrick et al., 2000). For example, mossy fiber synaptogenesis has been observed after kainate-induced epilepsy (Wuarin and Dudek, 1996) and kindling (Van-der-Zee et al., 1995). Moreover, it has been observed that high-frequency stimulation-inducing LTP produce mossy fiber synaptogenesis (Adams et al., 1997; Escobar et al., 1997). There is evidence suggesting that hippocampal synaptogenesis might occur in association with spatial memory formation. Becker et al. (1996) demonstrated that neural cell adhesion molecule polysialylation (NCAM-PSA), which allows structural plasticity, is necessary to improve performance of water maze task; also, changes

Received May 7, 2001; revised June 27, 2001; accepted June 27, 2001.

This work was supported by Milenio-CONACyT Grant 2000-2001 35806-N and DGAPA IN-214399. We acknowledge the technical assistance of Oreste Carbajal and Federico Jandete, and we give thanks to Shaun Harris and Dr. Guillaume Ferreira for the text review and to Yolanda Díaz de Castro for the preparation of this manuscript.

Correspondence should be addressed to Federico Bermúdez-Rattoni, Instituto de Fisiología Celular, Universidad Nacional Autónoma de México, Apartado Postal 70-253, 04510 México D. F., México. E-mail: fbermude@ifisiol.unam.mx.

Copyright (ㄷ) 2001 Society for Neuroscience $0270-6474 / 01 / 217340-09 \$ 15.00 / 0$ in the distribution of synapses were demonstrated in CA1 after the animals underwent Morris water maze training (Rusakov et al., 1997).

Recently, we found evidence suggesting that overtraining animals in a Morris water maze task induces mossy fiber synaptogenesis in CA3 stratum oriens using Timm's staining (RamírezAmaya et al., 1999). Timm's staining reveals the presence of zinc, and because this metal is highly concentrated in the mossy fiber boutons, the distribution of mossy fiber contacts can be observed by Timm's-stained granules using the light microscope. Mossy fiber projections to the CA3 region regularly establish their synaptic contacts in the stratum lucidum located in the apical region of the pyramidal cell layer. In the stratum oriens (basal region of the pyramidal cell layer) scarce mossy fiber synaptic contacts are found, so substantial increments of mossy fiber boutons to the stratum oriens can be considered as synaptogenesis (Ben-Ari and Represa, 1990). Furthermore, the induction of new synapses is observed mainly in animals that underwent several training sessions, suggesting that mossy fiber synaptogenesis could be related with spatial memory.

To establish the relevance of mossy fiber synaptogenesis for spatial memory, we evaluate long-term spatial memory performance of water maze-trained animals by testing them at 7 and $30 \mathrm{~d}$ after training. The performance of the animals in the memory test was correlated with Timm's staining observed in CA3 stratum oriens. To produce a pharmacological blockade of the spatial learning, the NMDA antagonist MK801 was applied before acquisition of water maze and immediately after, to test the possible effects of this drug on mossy fiber synaptogenesis using Timm's staining analysis. Furthermore, mossy fiber synaptogenesis distribution throughout the septotemporal axis of dorsal hippocampus was studied in serial hippocampal sections. 


\section{MATERIALS AND METHODS}

Subjects. Fifty-one Male Wistar Rats obtained from the "vivarium" of our Institute were individually caged in standard rat acrylic boxes and used for the experiment when within a weight range of 250-300 gm. All animals were kept in the same room with an average temperature of $22^{\circ} \mathrm{C}$, food and water was provided ad libitum, and animals were maintained under a inverted $12 \mathrm{hr}$ light/dark cycle, with lights on at 8:00 A.M.

Experimental groups. The animals were separated into five groups that underwent water maze training, and the last two were kept as controls. The first five groups of animals received one (WM1; $n=5$ ), two (WM2; $n=5$ ), three (WM3; $n=5$ ), four (WM4; $n=5$ ), and five (WM5; $n=$ 6 ) days of training. One group of swimming control was used (SC; $n=5$ ), in which the animals were introduced into the tank containing no platform, without illumination and with all spatial cues removed. The animals in this group were allowed to swim for $1 \mathrm{~min}$ and were introduced three times a day. This procedure was repeated for $5 \mathrm{~d}$, allowing them to swim a similar average distance as that of the trained animals. In the last group, the intact control (IC; $n=6)$ animals remained in their home cages for the entire experiment.

To evaluate the effects of intraperitoneal administration of the NMDA antagonist MK801 during and after acquisition of spatial learning as well as on mossy fiber synaptogenesis, two additional groups were used. These groups of animals underwent $5 \mathrm{~d}$ of training and received intraperitoneal injections of MK801 $(0.05 \mathrm{mg} / \mathrm{kg}$, diluted in $500 \mu \mathrm{l}$ of distillated water; Research Biochemicals, Natick, MA) either 15 min before (MKB; $n=7$ ) or immediately after (MKA; $n=7$ ) water maze training each day. One animal that presented motor problems (hyperactivity) during water maze task was discarded from the study of the group MKB. The W M5 animals from the first experiment were used for comparison analysis.

Water maze training. The behavioral task was performed in a water maze consisting of a circular pool tank measuring $1.50 \mathrm{~m}$ in diameter and $1.0 \mathrm{~m}$ high with a black floor and walls with a transparent acrylic platform located in a fixed position and submerged $1 \mathrm{~cm}$ under the water. The water maze tank is located in a separate dark room, illuminated by dim light, and surrounded by several spatial cues. The swimming paths of the animals were recorded by using a chromotrack system (San Diego Instruments). The training of the animals in the water maze consisted of 1 , $2,3,4$, or 5 identical daily sessions of 10 trials each. In each trial, the animal was introduced into the pool at different starting points for each trial up to a total of 10 trials per day (Nerad et al., 1996). The animal was allowed to swim for a maximum of $60 \mathrm{sec}$ or until it located the platform and was allowed to stand on the platform for $30 \mathrm{sec}$, after which the animal was placed in a waiting cage for $30 \mathrm{sec}$. If the animal did not find the platform within the time limit, it was led to it by hand. Most of the animals learned well in the first training session, but the asymptotic level for the variable (latency to the target) was not obtained until the third or fourth day session. Thus, any further training after the asymptotic level for latency scores was reached was considered as overtraining. Trained animals were tested in the tank 7 and $30 \mathrm{~d}$ after training; the test consisted of introducing the animals in to the tank using the first release point, with the platform removed. The animals were allowed to swim for 2 min. The path swum by the animals was recorded as described above, and the latency to the target, as well as the number of crossings over the target area, were used as dependent variables for analysis.

Timm staining. All animals were killed the next day after the last test session. The animals were put to sleep with an overdose of sodium pentobarbital (Sedal-Vet TM). Sodium sulfide solution (5.85 gm of NaS and $5.95 \mathrm{gm}$ of $\mathrm{NaH}_{2} \mathrm{PO}_{4}$ in $500 \mathrm{ml}$ of distillated water) was used as the first perfusion solution to remove blood ( $\sim 100-150 \mathrm{ml} /$ animal). Paraformaldehyde solution at $4 \%$ with $1 \%$ glutaraldehyde, $\mathrm{pH} 7.4$, was used as the fixative (two animals were lost because of bad sulfide solution perfusion; one from group WM1 and one from IC). The brains were removed from the skull and immersed in the fixative for 5-10 hr, after which the brains were immersed in $30 \%$ sucrose solution (diluted in PB, $\mathrm{pH}$ 7.4). Several days later, when the brains sank into the sucrose solution, $40-\mu \mathrm{m}$-thick slices of the completely dorsal hippocampus were serially obtained using a cryostat microtome. Timm's revealing method consists of submerging the tissue in a dense solution made of Arabic gum (60\% diluted 1:2 in distillated water), hydroquinone (30\% diluted 5.67 $\mathrm{gm} / 100 \mathrm{ml}$ in distillated water), and silver nitrate $(1 \%, 170 \mathrm{mg} / \mathrm{ml}$ in distillated water) in a $4.0 \mathrm{pH}\left(9 \%\right.$ of citrate buffer $12.75 \mathrm{gm}$ of $\mathrm{C}_{6} \mathrm{H}_{8} \mathrm{O}_{7}$ and $11.75 \mathrm{gm}$ of $\mathrm{C}_{6} \mathrm{H}_{5} \mathrm{O}_{7} \mathrm{Na}_{3}$ in $50 \mathrm{ml}$ of distilled water). The tissue was exposed for $\sim 1 \mathrm{hr}( \pm 15 \mathrm{~min})$. After the exposure, the tissue was run into an alcohol train (50,70,80, 90, 100\% diluted in distilled water) and isopropanol, terpineol, and xilol. The brain tissues were covered with

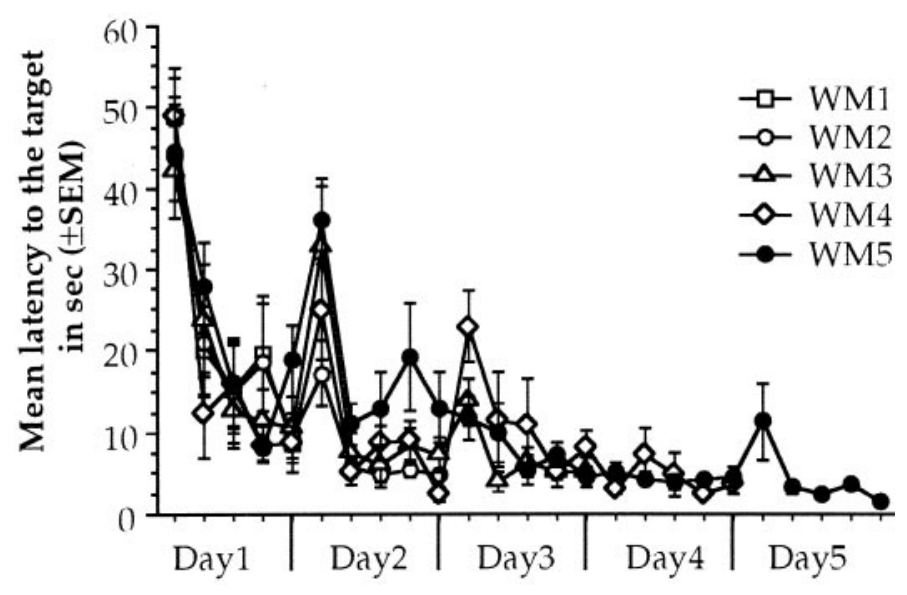

Figure 1. Water maze performance during acquisition of animals that received 1, 2, 3, 4, or $5 \mathrm{~d}$ of training. Each point is the average latency to the target for two consecutive trials that have the same average distance to the target area than any other pair of trials. Each day presents five pairs of trials.

crystal sheets using Canadian balsam. The presence of Timm's-stained granules in the stratum oriens was evaluated using image densitometry. The slices were carefully examined under light microscopy and classified into five different levels, ranging from the most anterior (1) to the most posterior (5) dorsal hippocampus using the morphological features of the dentate gyrus as reference (see Fig. 6). Six coronal sections from the dorsal hippocampus were selected: three from level 2, and three from level 3 (see Figs. 6 and 10). The stratum oriens of this section was digitized by a camera (model c2400; Hamamatsu, Tokyo, Japan) connected to an optical microscope (labophot-2; Nikon, Tokyo, Japan), after which they were equalized by the application of the same contrast and brightness parameters (Adobe Photoshop 5.0), and then analyzed by using image analysis software (Scion Image; Scion Corporation, Frederick, MD). The surface area with an optical density range between 120 and 255 points in a black and white scale (the same range of Timm's staining in the stratum lucidum) occupied by Timm's granules in the stratum oriens, was measured in each image, so each animal brain obtained six right and six left measures. The mean area from these 12 measures was obtained for each animal to compare the different groups.

Statistical analysis. Repeated measures ANOVA, one-way ANOVA with Fisher post hoc, and Pearson's correlation were used as appropriate.

\section{RESULTS}

\section{Overtrained animals performed better in memory test}

Repeated measures ANOVA of the latency to reach the platform during water maze acquisition showed significant differences through trials in day $1\left(F_{(9,36)}=23.443 ; p<0.001\right)$, day 2 $\left(F_{(9,27)}=18.259 ; p<0.001\right)$, and day $3\left(F_{(9,18)}=6.802 ; p<0.01\right)$ and no significant difference among groups with no interaction in all trained groups. Finally, there were no differences through trials for the last two days (days 4 and 5) with no group differences and no interaction. Differences through trials are considered as the learning curve and imply that trained animals improve performance in that session. When performance of the animals is stabilized in the asymptotic level, further training is considered as overtraining, so WM4 and WM5 animals are the overtrained groups (Fig. 1).

Water maze test results showed that there were significant differences between groups in the latency $\left(F_{(5,25)}=9.750 ; p<\right.$ $0.01)$ as well as in the number of crossings through the target area at $7 \mathrm{~d}$ after training $\left(F_{(5,25)}=16.616 ; p<0.01\right)$, revealing that the amount of training is an important factor determining memory performance $7 \mathrm{~d}$ after acquisition (Fig. 2). Post hoc Fisher analysis of the latency to reach the target showed significant differ- 

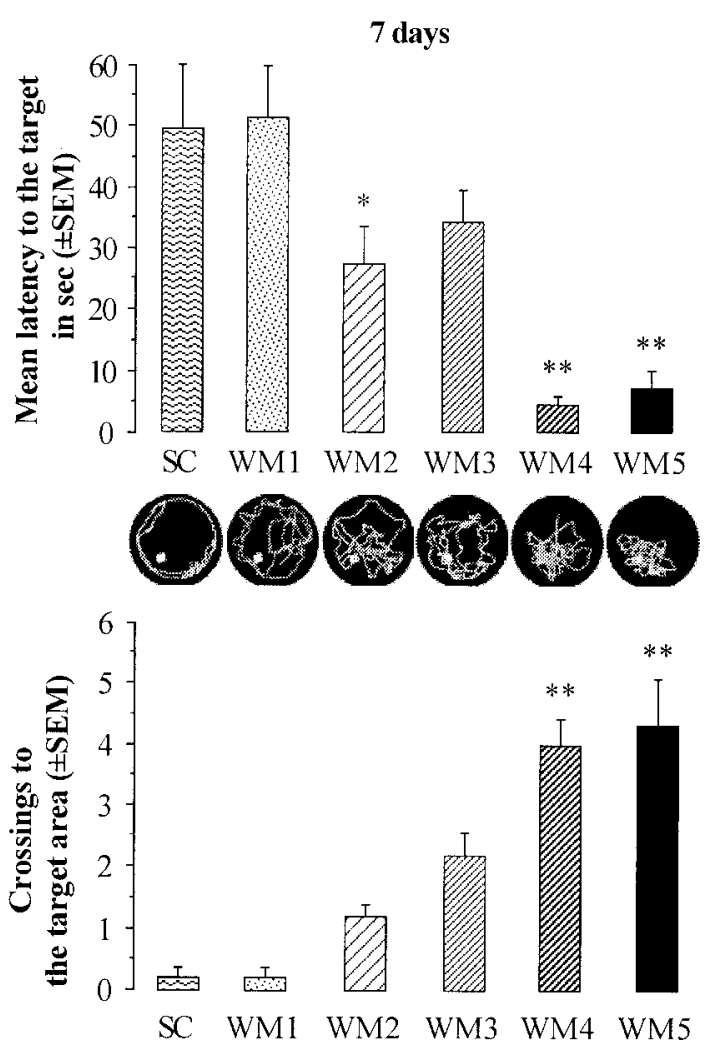

Figure 2. Memory test scores at $7 \mathrm{~d}$ after acquisition. The top of the figure shows latency to the target of trained animals for $1,2,3,4$, and $5 \mathrm{~d}$ $(W M)$ and swimming control animals $(S C)$. The middle shows representative swimming paths during 1 min test without the platform. Bottom, The number of crossings over the target are during 1 min test. ${ }^{*} p<0.05$, ${ }^{* *} p<0.01$ compared with the SC.

ences in WM4 and WM5 when compared with the rest of the groups in the test done $7 \mathrm{~d}$ after training ( $p$ values $<0.01$ ), and the group trained for $2 \mathrm{~d}$ showed significant differences when compared with the SCs ( $p$ values $<0.05$ ). For the number of crossings, post hoc analysis revealed that WM5 and WM4 groups crossed the target area significantly more than the rest of the groups ( $p$ values $<0.01)$. When the animals were tested $30 \mathrm{~d}$ after training (Fig. 3), simple ANOVA revealed significant differences between groups in the latency $\left(F_{(5,25)}=2.805 ; p<0.05\right)$ and in the number of crossing to the target area $\left(F_{(5,25)}=6.170\right.$; $p<0.01)$. Post hoc analysis on the latency revealed that only WM4 presented differences when compared with the SC, WM1, and WM2 groups ( $p$ values $<0.05$ ). For the number of crossings, WM5 and WM4 showed significant differences compared with the SC ( $p<0.01$ and $p<0.05$, respectively). This data reveals that animals trained for 4 and $5 \mathrm{~d}$ recall the spatial location of the platform significantly better than the rest of the groups.

\section{NMDA antagonist blocked acquisition of spatial task}

As can be seen in Figure 4, repeated measures ANOVA done on latency to the target during water maze acquisition of MK801treated animals showed significant group differences during the first $4 \mathrm{~d}$; day $1(F(2,17)=20.922 ; p<0.01)$, day $2\left(F_{(2,17)}=6.103\right.$; $p<0.05)$, day $3\left(F_{(2,17)}=14.407 ; p<0.01\right)$, and day $4\left(F_{(2,17)}=\right.$ $19.795 ; p<0.01)$. Also, there were differences through trials for all the days; day $1\left(F_{(9,18)}=8.999 ; p<0.01\right)$, day $2\left(F_{(9,18)}=\right.$ $7.226 ; p<0.01)$, day $3\left(F_{(9,18)}=4.519 ; p<0.01\right)$, day $4\left(F_{(9,18)}=\right.$ 3.946; $p<0.01)$, and day $5\left(F_{(9,18)}=2.441 ; p<0.05\right)$ with no
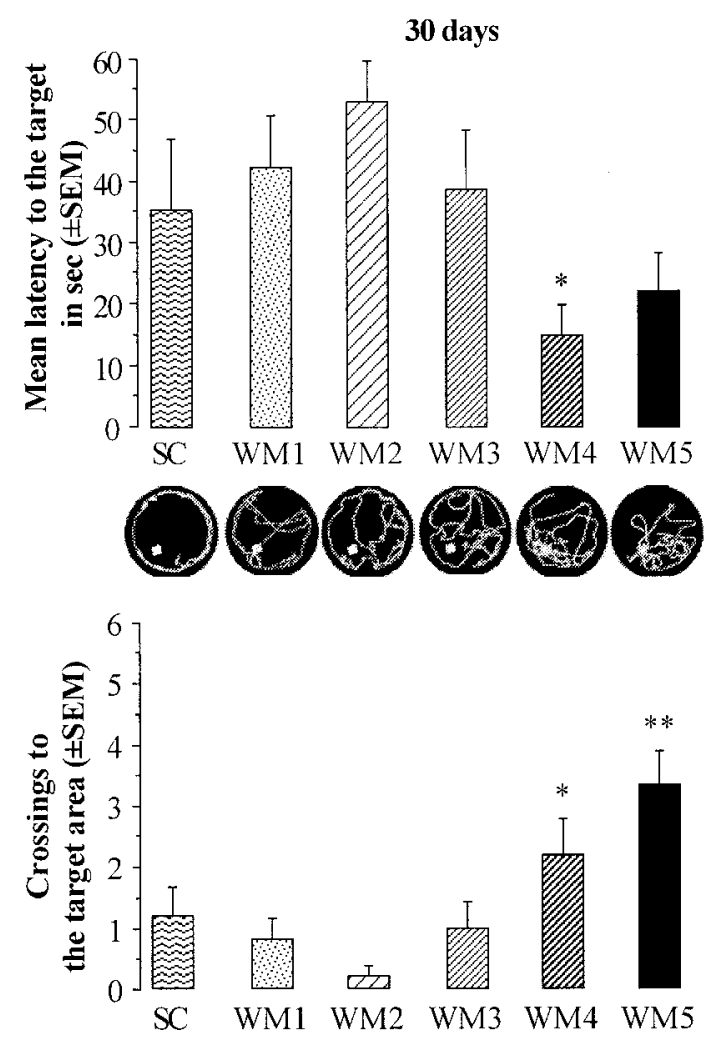

Figure 3. Memory test scores at $30 \mathrm{~d}$ after acquisition. The top of the figure shows latency to the target of trained animals for 1, 2, 3, 4, and $5 \mathrm{~d}$ $(W M)$ and swimming control animals $(S C)$. The middle shows representative swimming paths during 1 min test without the platform. Bottom, The number of crossings over the target are during 1 min test. ${ }^{*} p<0.05$, $* * p<0.01$ compared with the SC.

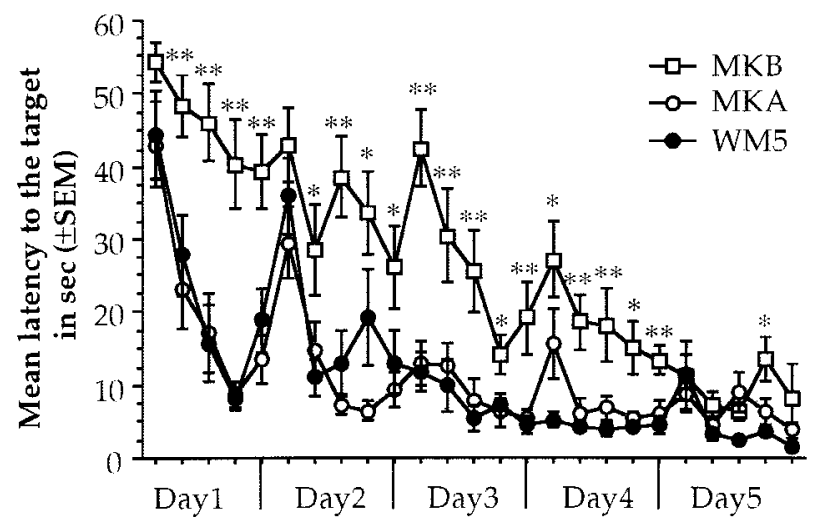

Figure 4. Water maze performance during acquisition of animals that received $5 \mathrm{~d}$ of training (WM5) and animals with the same amount of trained but treated $15 \mathrm{~min}$ before $(M K B)$ or immediately after $(M K A)$ with $0.05 \mathrm{mg} / \mathrm{kg}$ of M K801. Each point is the average latency to the target for two consecutive trials that have the same average distance to the target area than any other pair of trials. Each day presents five pairs of trials. ${ }^{*} p<0.05, * * p<0.01$ compared with WM5.

significant interaction. The animals in which MK801 was administered before acquisition (MKB) presented an impaired acquisition during the first $2 \mathrm{~d}$ because there were no significant learning curves at day $1\left(F_{(6,9)}=1.051 ; p=0.4134\right)$ and day 2 $\left(F_{(6,9)}=1.779 ; p=0.0938\right)$. At days 3 and 4 , the latency to the target showed statistical differences through trials $\left(F_{(6,9)}=2.560\right.$; $p<0.05$ and $F_{(6,9)}=3.237 ; p<0.01$, respectively), indicating that 


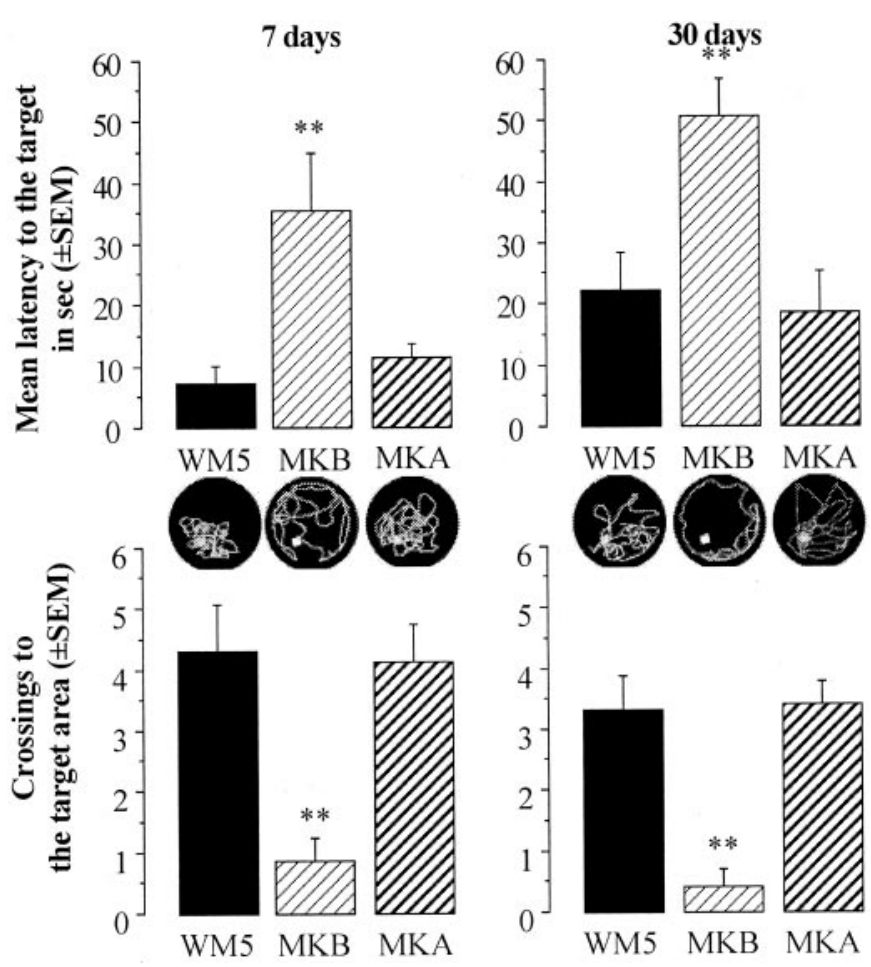

Figure 5. Memory test scores at 7 (left) and 30 (right) d after acquisition. The top of the figure shows latency to the target from $5 \mathrm{~d}$-trained animals (WM5) and $5 \mathrm{~d}$-trained animals receiving MK801, 15 min before $(M K B)$ or immediately after $(M K A)$ water maze training. The middle shows representative swimming paths during 1 min test without the platform. The bottom shows the number of crossings over the target are during 1 min test. ${ }^{*} p<0.05,{ }^{* *} p<0.01$ compared with WM5.

in those days learning occurred in MKB animals. Nevertheless, the group differences remained, revealing a disrupted performance on water maze training by the administration of MK801 before acquisition. At day 5, there were no more group differences $\left(F_{(2,17)}=2.276 ; p=0.13\right)$, and MKB animals reached the asymptotic level of performance in the water maze task. Conversely, MK801 administered after acquisition (MKA) did not affect consolidation of the spatial task. MKA animals showed significant decreased latencies to reach the target through trials at day $1\left(F_{(6,9)}=5.445 ; p<0.01\right)$ and day $2\left(F_{(6,9)}=5.288 ; p<\right.$ $0.01)$; no differences were found from days $3-5$. This indicates that the MKA animals reach the asymptotic level of performance at day 3, showing no effect of MK801 administration in spatial memory consolidation.

In the memory test done $7 \mathrm{~d}$ after training, (Fig. 5) simple ANOVA showed significant group differences in the latency $\left(F_{(2,17)}=6.185 ; p<0.01\right)$ and the number of crossings to the target area $\left(F_{(2,17)}=10.748 ; p<0.01\right)$. At $30 \mathrm{~d}$ after training significant differences were found in the latency $\left(F_{(2,17)}=7.518\right.$; $p<0.01)$ and the number of crossing to the target area $\left(F_{(2,17)}=\right.$ 18.026; $p<0.01)$. The MK801 applied before the acquisition clearly disrupts water maze memory test (Fig. 5). The animals from MKB group showed significantly less crossings and longer latency to the target area at 7 or $30 \mathrm{~d}$ after training compared with MKA and WM5 groups ( $p$ values $<0.01)$. This evidence revealed that MK801 administration disrupts acquisition and retention of the water maze task. Finally, when MK801 was applied after the learning trials, the memory scores remained intact indicating that MK801 applied after acquisition did not affect retention of the spatial task.

\section{Mossy fiber synaptogenesis correlates with spatial memory performance}

As previously reported, Timm's staining revealed mossy fiber synaptogenesis in the stratum oriens of animals that were overtrained in the Morris water maze, whereas the stratum oriens of swimming and intact control animals presented scarce Timm'sstained granules (Fig. 6). Simple ANOVA on the average of Timm's-positive surface area determined by quantitative densitometry analysis (Fig. 7) revealed significant differences between groups $\left(F_{(6.28)}=8.031 ; p<0.01\right)$. Animals in groups WM4 and WM5 presented significantly more stained area in the stratum oriens when compared with controls (IC, SC, $p$ values $<0.01$ ) and when compared with the other trained groups (WM1, WM2, $p$ values $<0.01$; WM3, $p<0.05$, respectively). No statistical difference was observed between WM4 and WM5. This data suggest that mossy fiber synaptogenesis occurred mainly in the animals that were overtrained in the Morris water maze for 4 or $5 \mathrm{~d}$ and not in animals that received less training (WM3, WM2, and WM1) or no spatial training at all (SC and IC).

To establish if mossy fiber synaptogenesis can be related with memory performance, Pearson's correlation was performed between the average of Timm's-stained area and the latency, or the number of crossings over the target area during the test days. Only the trained animals were included in this analysis (WM1, WM2, WM3, WM4, and WM5). The analysis revealed a significant negative correlation between the latency to the target and Timm's staining area in the stratum oriens in the test done at $7 \mathrm{~d}$ $(-0.617 ; p<0.01)$ but not at $30 \mathrm{~d}$ after training. Furthermore, the number of crossings to the target area and the Timm's-stained area showed positive correlation for the test done at $7 \mathrm{~d}(C=$ $0.566 ; p<0.01)$ and $30 \mathrm{~d}(C=0.564 ; p<0.01)$ after training. This evidence suggests a functional relation between mossy fiber synaptogenesis and spatial memory performance.

\section{NMDA antagonists blocked synaptogenesis}

Simple ANOVA on mossy fiber staining analysis revealed significant differences between groups $\left(F_{(4,24)}=7.392 ; p<0.01\right)$. Post hoc Fisher analysis showed that MKB animals presented significantly less mossy fiber stained area in the stratum oriens when compared with WM5 group ( $p<0.01$ ), and no significant differences were found with SC or IC groups (Fig. 8). The analysis of the mossy fiber-stained area from MKA animals showed significant differences compared with SC and IC groups ( $p$ values $<$ 0.01 , respectively) and not with WM5 group. Pearson's correlation analysis using WM5, MKB, and MKA groups showed a significant negative correlation between Timm's-stained area in the stratum oriens and the latency to the target in the test done at $7 \mathrm{~d}(C=0.533 ; p<0.05)$ but not at $30 \mathrm{~d}$ after training. Significant correlation was also found with the number of crossings in the test done 1 week $(C=0.639 ; p<0.01)$ and 1 month after training $(C=0.530 ; p<0.05)$. These results indicate that pharmacological blockade of learning affects the behavioral induction of mossy fiber synaptogenesis in CA3 stratum oriens, as well as memory performance. These findings further support the idea that this morphological change may be related to spatial memory.

\section{Synaptogenesis is observed mainly in the more septal area of dorsal hippocampus}

To understand the distribution of mossy fiber synaptogenesis throughout the rat hippocampus, the Timm's-stained area was 
A Dorsal

Right

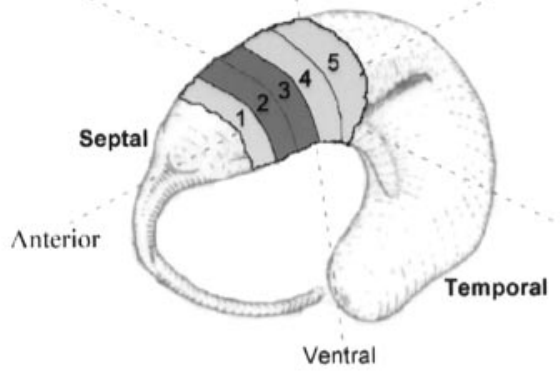

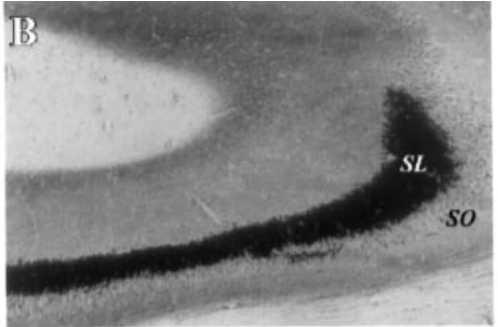

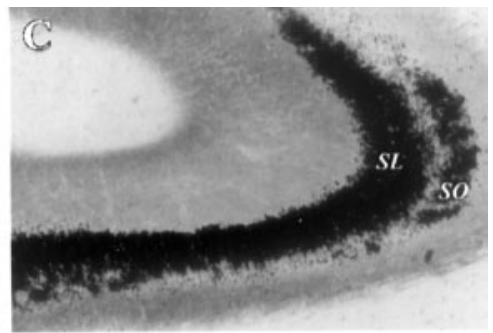

Left
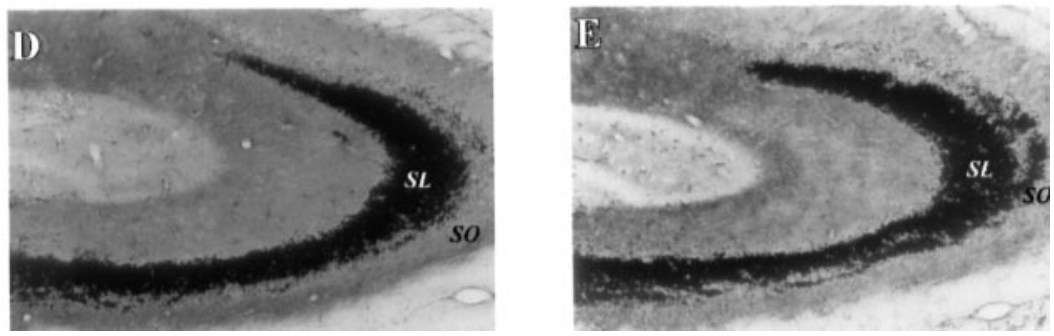

Figure 6. A, Schematic representation of the left hippocampus showing in the gray subdivisions, the area obtained for Timm's staining. Regions 1, 4, and 5 (clear gray) were stained for Timm's but not used for density analysis, the first three brain slices from region 2 and the first three from region 3 (dark gray) were used for the computer-assisted Timm's-stained analysis in the stratum oriens. Photomicrographs taken at $4 \times$ from region 2 of CA3 hippocampus stained for Timm's of representative swimming control animal $(S C)(B), 5 \mathrm{~d}$ water maze-trained $(W M 5)(C)$, 5 d-trained treated with MK801 before $(M K B)(D)$, and trained treated with MK801 after $(M K A)(E)$.

A)

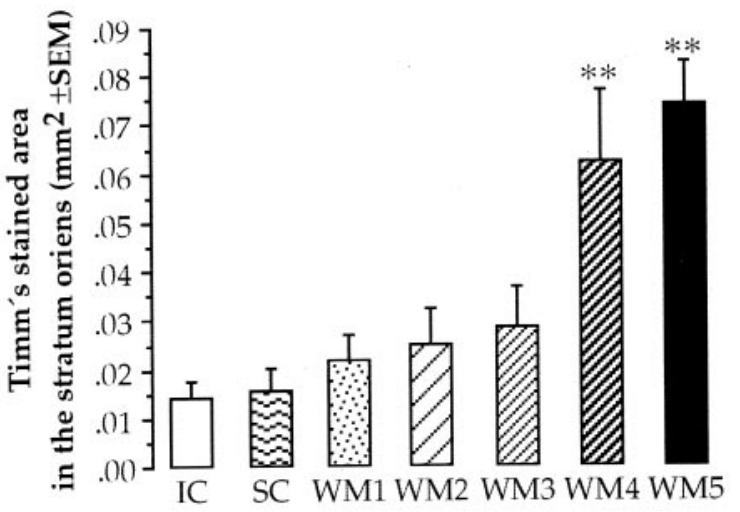

B)

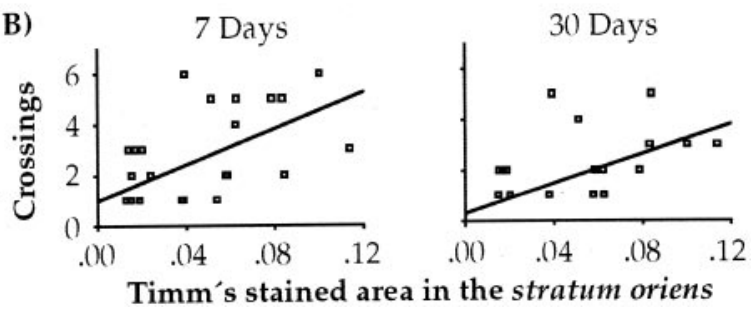

Figure 7. A, Average Timm's-stained area in the stratum oriens of animals trained for $1,2,3,4$, and $5 \mathrm{~d}$ (WM1,WM2,WM3,WM4, and $W M 5$, respectively), swimming controls $(S C)$, and intact control animals (IC). **p $<0.01$ compared with SC. B, Scattergram plot between synaptogenesis and memory performance at 7 and $30 \mathrm{~d}$ after training. All groups included in the correlation except SC and IC.

analyzed by the septotemporal axis from which the image was taken. Repeated measures ANOVA of the Timm's-stained area was done in SC, MKB, MKA, WM1, WM3, and WM5 groups. Because no significant differences were found between left and right stratum oriens, the mean of right and left stratum oriens was used for this analysis. The analysis revealed significant differences
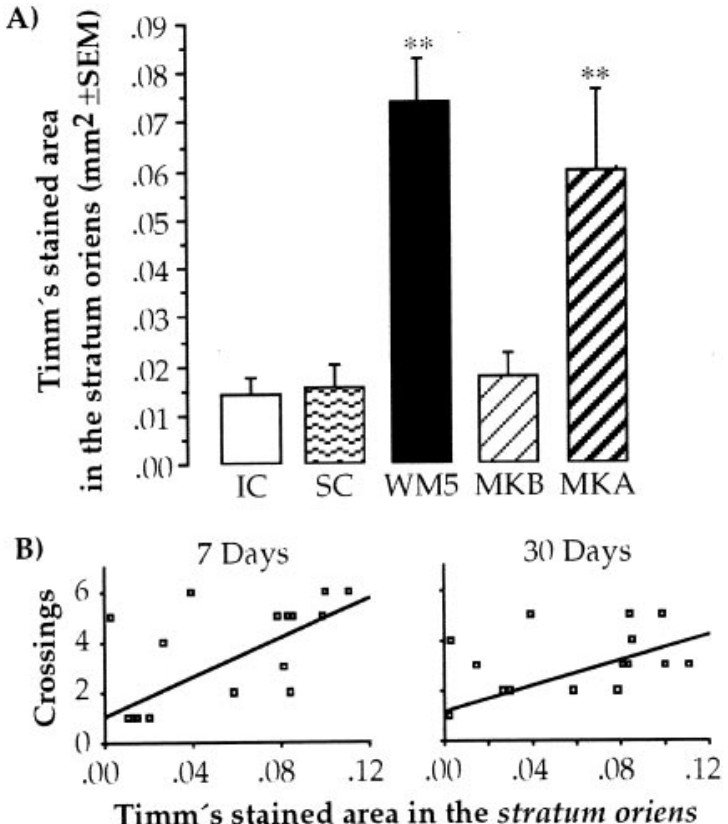

Figure 8. A, Average Timm's-stained area in the stratum oriens of animals trained for $5 \mathrm{~d}$ (WM5), animals trained for $5 \mathrm{~d}$ and treated with MK801 before $(M K B)$ and after acquisition $(M K A)$, swimming controls $(S C)$, and intact control animals $(I C) .{ }^{* *} p<0.01$ compared with SC. $B$, Scattergram plot between synaptogenesis and memory performance at 7 and $30 \mathrm{~d}$ after training. All groups included in the correlation except SC and IC.

among groups $\left(F_{(5,27)}=5.606 ; p<0.01\right)$, between septotemporal measures $\left(F_{(11,55)}=5.766 ; p<0.01\right)$ with no significant interaction. This indicates that the Timm's-stained area in the stratum oriens decrease throughout the septotemporal measures (Fig. 9) and that the animals that underwent water maze overtraining presented more Timm's-stained granules than the control groups, so the presumed synaptogenesis is observed mainly in the septal 


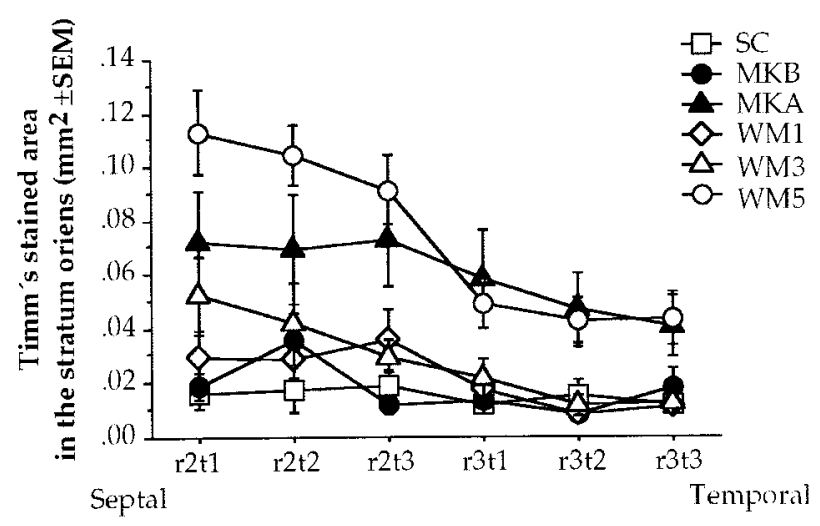

Figure 9. Average Timm's-stained area in the stratum oriens throughout the septotemporal axis of regions $2(r 2)$ and $3(r 3)$ with the tissues taken for analysis $(t 1-3)$ of animals trained for 1,3 , and $5 \mathrm{~d}(W M)$, trained animals for $5 \mathrm{~d}$ treated with MK801 before $(M K B)$ and after acquisition $(M K A)$, and the swimming controls $(S C)$.

portion of dorsal hippocampus and gradually decrease toward the temporal pole. Also, as can be seen in Figure 9, the animals administered with MK801 before acquisition clearly showed poor Timm's staining in the stratum oriens throughout the hippocampal septotemporal axis, whereas the animals administered with MK801 after acquisition still showed Timm's staining in a similar portion and in a similar extent of dorsal hippocampus than the overtrained animals.

The Timm's staining results can be observed in more detail in Figure 10, where Adobe Photoshop-processed photomicrographs of representative control and overtrained animals are shown throughout the septotemporal axis of dorsal CA3 hippocampus stained by Timm's. It is clear that in the control animal scarce staining is observed in the stratum oriens, whereas in the overtrained animal staining is evident in this region, abundantly at level 2 and decreased at level 3, with scarce staining at level 4, and almost absent at level 5 (Fig. 10). In summary, the largest area stained by Timm's in CA3 stratum oriens is observed in the more septal region of the dorsal hippocampus, and the staining decreased gradually toward the temporal region selected for the analysis, suggesting that this morphological change occurs in the more septal region of hippocampal CA3 stratum oriens.

\section{DISCUSSION}

\section{Is mossy fiber synaptogenesis related to spatial memory formation?}

The results from these experiments revealed that water maze overtraining produces a remarkable improvement in a memory test done 1 week and 1 month after training. In a previous report we showed that spatial overtraining could induce synaptogenesis in CA3 stratum oriens (Ramírez-Amaya et al., 1999). In the present experiments, we found a substantial increase of mossy fiber projection to the stratum oriens in animals that received overtraining in the Morris water maze, and their performance in the memory tests positively correlates with this, suggesting a functional correlation between synaptogenesis and spatial memory formation.

It has been proposed that the mossy fiber system modulates the activity of CA3 cells in which plastic events will allow information to be encoded (McClelland and Goddard, 1996). In accordance with this idea, destruction of dentate gyrus cells, and hence mossy fiber projections, disrupts acquisition and retention of water maze task (McNaughton et al., 1989; Xavier et al., 1999). Mizumori et al. (1999) proposed that the specific contribution of the hilar/CA3 region is to compare the expected spatial context with that currently being experienced, supporting the idea that synaptic plasticity in the mossy fiber projection to CA3 may be involved in the storage of spatial representations (McNaughton and Morris 1987; McClelland and Goddard, 1996). Mossy fiber projections regularly reach the stratum lucidum and the newly formed mossy fibers project to the stratum oriens. Although we found evidence of synaptogenesis in the stratum oriens, stratum lucidum synaptogenesis cannot be discarded. The functional significance of mossy fiber projections to the stratum oriens is currently unknown. However, it is possible that these projections have a stronger influence over pyramidal cell firing because of their proximity to the axon hillock, or it can modulate CA3 by establishing synaptic contacts with inhibitory cells located in the stratum oriens (Oliva et al., 2000; Poncer et al. 2000).

An increased survival of new neurons in the dentate gyrus that are likely to project to CA3 cells has been demonstrated after spatial training (Kuhn et al., 1996; Kempermann et al., 1997; Hastings and Gould, 1999). Recently, Elizabeth Gould's group (Shors et al., 2001) showed that inhibition of neurogenesis in the rat impairs hippocampal trace conditioning. Thus, it is plausible to think that neurogenesis in the dentate gyrus may be related to the storage of spatial representations and perhaps to synaptogenesis in CA3. Preliminary results in our laboratory showed that specific lesions of CA3 region disrupt acquisition of water maze and clearly impair memory performance even when lesions were made 2 weeks after acquisition. This evidence suggests that CA3 might be necessary for long-term spatial information storage in which synaptic plasticity, including morphological changes in the dentate gyrus projection to CA3, may underlie memory formation. Further research should be conducted to establish if mossy fiber synaptogenesis is reversible and if this reversibility might or not might be correlated with forgetting.

Another possibility is that these new mossy fiber projections are responsible for improving the overall spatial abilities of rats. Moser et al. (1994) observed that dendrite branching induced by a complex environment correlates with improved water maze performance. Previous studies using Timm's staining analysis have suggested the relevance of mossy fiber projection to CA3 infrapyramidal layer for spatial learned behaviors. It has been observed that animals from different strains or close species occupying different habitats presented different mossy fiber projections to the infrapyramidal layer, and the animals with more abundant mossy fibers projections to the infrapyramidal layer presented a better performance on spatial-related tasks (Schwegler et al., 1993; Bernasconi-Guastalla et al., 1994; Pleskacheva et al., 2000). They propose that genetic differences might account for the anatomical and behavioral variability, however, the observation that close species occupying different habitats also present such anatomical and behavioral differences suggest that the environment may also be a very important factor for inducing the morphological differences in mossy fiber projection. In this regard, the differences in mossy fiber projection found in these works should not only be attributed to inherited variables, but to how experience can promote plastic events in the hippocampus of the different species (Schwegler et al., 1993; Bernasconi-Guastalla et al., 1994). Because experience induces morphological changes, animals living in enriched conditions (Moser et al., 1994) or a 


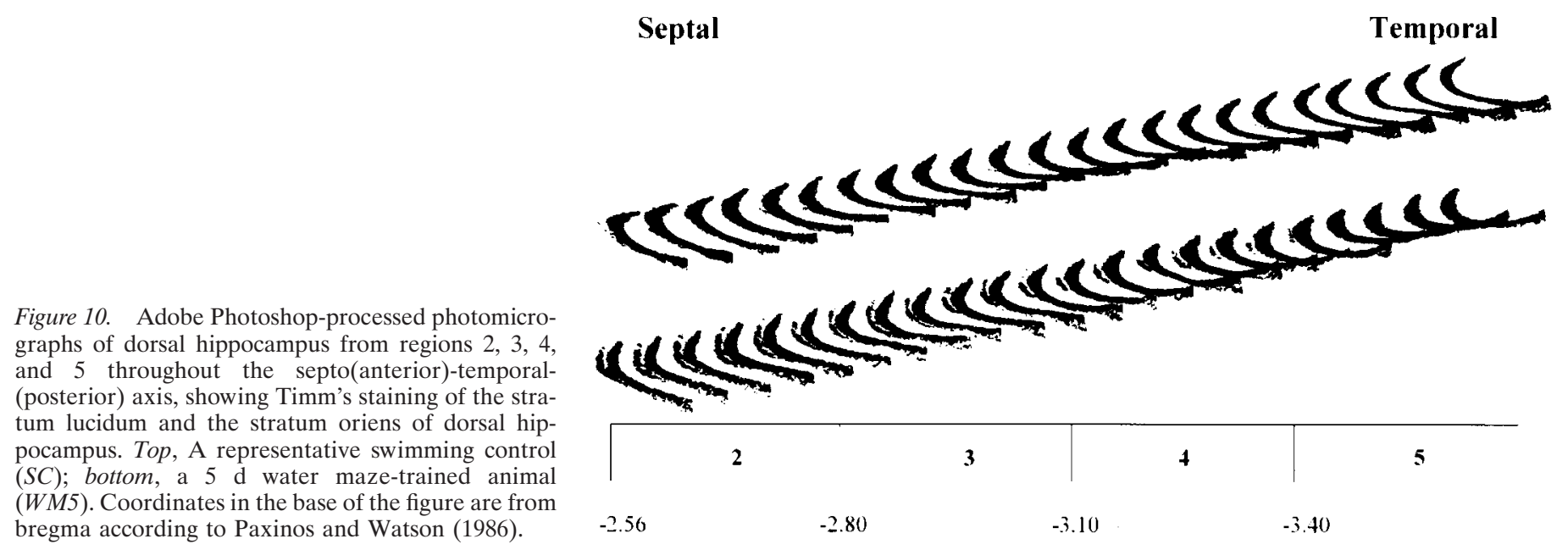

favorable environment (Pleskacheva et al., 2000) will present different anatomical and behavioral abilities when compared with animals living in a restricted environment as laboratory rats. In the case of laboratory rats, it is possible that the mossy fiber pathway is underutilized because the stimulation is restricted, and when confronted with any spatial behavioral challenge, the modulatory capacity of the mossy fiber pathway may be modified through synaptogenesis.

\section{Pharmacological blockade of learning blocks mossy fiber synaptogenesis}

The data obtained herein showed that administration of 0.05 $\mathrm{mg} / \mathrm{kg}$ of MK801 before training clearly disrupts performance of water maze acquisition, as has been observed previously (Åhlander et al., 1999). Although the animals overcome the impairing effect of MK801 after $4 \mathrm{~d}$ of training, these animals cannot be considered overtrained, and accordingly, their performance in the memory tests was very poor at 7 and $30 \mathrm{~d}$ after training, which is consistent with the idea that overtraining is responsible for improving performance in the memory test. Moreover, the animals treated with MK801 before acquisition did not show an increase of mossy fiber projections to the stratum oriens, even though these animals underwent the same training conditions as the animals in WM5 group, supporting the idea that overtraining is the factor responsible for the induction of synaptogenesis. Conversely, the administration of MK801 after acquisition did not affect spatial learning consolidation as observed previously (McLamb et al., 1990). Thus, no effects were observed in the memory test done 7 and $30 \mathrm{~d}$ after training, indicating that animals administered with MK801 after acquisition consolidate normally. Furthermore, mossy fiber synaptogenesis was observed in these animals, even under the effects of NMDA receptor antagonist.

The involvement of NMDA receptors in learning and memory has long been suggested by their role in LTD, LTP (Malenka, 1994; Malenka and Nicoll, 1999), and in spatial learning (Martinez and Derrick, 1996). The evidence obtained here is consistent with previous literature, suggesting the involvement of glutamatergic neurotransmission in spatial learning, underlying plastic events such as LTP, and probably synaptogenesis (Butler et al., 1999). In this regard, NMDA receptors have also been implicated in the establishment of new synapses (Friedman et al., 2000). Although the MK801 dosage used in this experiment did not produce important motor effects to explain NMDA blockade of spatial learning (Åhlander et al., 1999), it cannot be certainly established if mossy fiber synaptogenesis is dependent on NMDA-related mechanisms.

The proper characterization of the molecular mechanisms involved in mossy fiber synaptogenesis might require a broader study of the effects of different receptor antagonists, as well as the expression of different molecules involved in the induction, guidance, and stabilization of mossy fiber synapses. In this work, the purpose of inactivating NMDA receptors was to understand if a pharmacological blockade of learning was enough to disrupt synaptogenesis. The main differences between MKA and MKB are that learning was allowed to occur in MKA group, whereas the learning ability of MKB animals was impaired. Accordingly, memory performance was impaired in MKB animals, but not in MKA group, further supporting the idea that overtraining improves spatial memory performance, which might be related to mossy fiber synaptogenesis.

\section{Synaptogenesis occurs in the more septal area of the dorsal hippocampus}

In this experiment we also found evidence suggesting that increased mossy fiber projection to CA3 occurs mainly in the more septal region of the dorsal hippocampus. The lamellar hypothesis of hippocampal organization is no longer accepted, instead a more complex organization with differences throughout the septotemporal and proximodistal axis of hippocampus is now evident (Amaral and Witter, 1989). Ishizuka et al. (1990) found that projections from CA3 that innervates CA3 and CA1 are different throughout the septotemporal poles and also throughout the proximodistal axis. The bidirectional projections of hippocampus with the septum is well known to be different throughout the septal and temporal poles (Nyakas et al., 1987; Gaykema et al., 1991). Also, it has been demonstrated that the entorhinal cortex and amygdala projects differently through this axis (Ino et al., 1998; Pikkarainen et al., 1999).

These anatomical differences might have functional implications for the different regions of the hippocampus. Accordingly, it has been established that only dorsal but not ventral hippocampal lesions produce behavioral impairment of spatial-related tasks (Moser and Moser, 1998; Bannerman et al., 1999). Operant behavior-induced c-Fos expression has been observed to occur mainly in the dorsal hippocampus (Bertaina-Anglade et al., 
2000). Rapp et al. (1999) studied the hippocampal projections between young and aged animals, observing that the septal region of mossy fiber projection was altered in senescent animals, presenting deficits in performance of spatial-related tasks. Our data are consistent with these observations; because our analysis was made only in the dorsal hippocampus, we cannot be certain if similar synaptogenesis is occurring in the ventral hippocampus. However, the evidence presented here might suggest different roles of the septal and temporal parts of the dorsal hippocampus in which the septal pole is suggested to be of great relevance for spatial memory formation.

\section{Conclusions}

The data obtained here support the idea that learning may promote morphological plastic events in the CNS, particularly synaptogenesis, which in the hippocampus can be observed after spatial overtraining in the mossy fiber projection to CA3. Although the functional significance of the new mossy fibers is not yet clear, the data obtained here suggest that these new mossy fibers may be related with memory formation, which is consistent with the idea that CA3 hippocampus is important for storing spatial representations. In this regard, we hypothesize that synaptogenesis might be responsible for producing long-lasting changes in the representation of spatial stimuli. Furthermore, NMDA dependent mechanisms are responsible for spatial memory formation and perhaps might be related to synaptogenesis. Because this form of morphological plasticity was observed mainly in the septal side of the dorsal hippocampus, it is possible that in this particular region, long-lasting plastic changes may underlie the establishment of long-term spatial memory.

\section{REFERENCES}

Åhlander M, Misane I, Schöt PA, Ögren SO (1999) A behavioural analysis of the spatial learning deficit induced by the NMDA receptor antagonist MK801 (dizocilpine) in the rat. Neuropsychopharmacology $21: 414-426$.

Adams B, Lee M, Fahnestock M, Racine RJ (1997) Long-term potentiation trains induce mossy fiber sprouting. Brain Res 775:193-197.

Amaral DG, Witter MP (1989) The three-dimensional organization of the hippocampal formation: a review of anatomical data. Neuroscience 31:571-591.

Bannerman DM, Yee BK, Good MA, Heupel MJ, Iversen SD, Rawlins JN (1999) Double dissociation of function within the hippocampus: a comparison of dorsal, ventral, and complete hippocampal cytotoxic lesions. Behav Neurosci 113:1170-1188.

Bear MF (1996) A synaptic basis for memory storage in the cerebral cortex. Proc Natl Acad Sci USA 93:13453-13459.

Becker CG, Artola A, Gerardy-Schahn R, Becker T, Welzl H, Schachner M (1996) The polysialic acid modification of the neural cell adhesion molecules is involved in spatial learning and hippocampal long-term potentiation. J Neurosci Res 45:143-152.

Ben-Ari Y, Represa A (1990) Brief seizures episodes induce long-term potentiation and mossy fiber sprouting in the hippocampus. Trends Neurosci 13:312-318.

Bernasconi-Guastalla S, Wolfer DP, Lipp HP (1994) Hippocampal mossy fibers and swimming navigation in mice: correlations with size and left-right asymmetries. Hippocampus 4:53-64.

Bertaina-Anglade V, Tramu G, Destrade C (2000) Differential learningstage dependent patterns of c-Fos protein expression in brain regions during the acquisition and memory consolidation of an operant task in mice. Eur J Neurosci 12:3803-3812.

Butler AK, Uryu K, Rougon G, Chesselet MF (1999) N-Methyl-DAspartate receptor blockade affects polysialylated neural cell adhesion molecule expression and synaptic density during striatal development. Neurosci 89:1169-1181.

Derrick BE, York AD, Martinez JL Jr (2000) Increased granule cell neurogenesis in the adult dentate gyrus following mossy fiber stimulation induce long-term potentiation. Brain Res 857:300-307.

Dudai Y (1989) Some basic notions and their ontogenesis. In: The neurobiology of memory: concepts, findings, trends, Chap 1, pp 3-18. New York: Oxford UP.
Escobar ML, Barea-Rodriguez EJ, Derrick BE, Reyes JA, Martinez JL Jr (1997) Opioid receptor modulation of mossy fiber synaptogenesis: independence from long-term potentiation. Brain Res 751:330-335.

Friedman HV, Bresier T, Garner CC, Ziv NE (2000) Assembly of new individual excitatory synapses: time course and temporal order of synaptic molecule recruitment. Neuron 27:57-69.

Gaykema RP, van der Kuil J, Hersh LB, Luiten PG (1991) Patterns of direct projections from the hippocampus to the medial septum-diagonal band complex: anterograde tracing with Phaseolus vulgaris leucoagglutinin combined with immunohistochemistry of choline acetyltransferase. Neuroscience 43:349-360.

Hastings NB, Gould E (1999) Rapid extension of axons into the CA3 region by adult-generated granule cells. J Comp Neurol 413:146-154.

Ino T, Kaneko T, Mizuno N (1998) Direct projections from the entorhinal cortical layers to the dentate gyrus, hippocampus, and subicular complex in the cat. Neurosci Res 32:241-265.

Ishizuka N, Weber J, Amaral DG (1990) Organization of intrahippocampal projections originating from CA3 pyramidal cells in the rat. J Comp Neurol 295:580-623.

Kempermann G, Kuhn HG, Gage FH (1997) More hippocampal neurons in adult mice living in an enriched environment. Nature 386:493-495.

Klintsova AY, Greenough WT (1999) Synaptic plasticity in cortical systems. Curr Opin Neurobiol 9:203-208.

Kuhn HG, Dickinson-Anson H, Gage FH (1996) Neurogenesis in the dentate gyrus of the adult rat: Age-related decrease of neuronal progenitor proliferation. J Neurosci 16:2027-2033.

Malenka RC (1994) Synaptic plasticity in the hippocampus LTP and LTD. Cell 78:535-538.

Malenka RC, Nicoll RA (1999) Long-term potentiation-a decade of progress. Science 285:1870-1874.

Martinez Jr JL, Derrick BE (1996) Long-term potentiation and learning. Annu Rev Psychol 47:173-203.

McClelland JL, Goddard NH (1996) Considerations arising from a complementary learning systems perspective on hippocampus and neocortex. Hippocampus 6:654-665.

McLamb RL, Williams LR, Nanry KP, Wilson WA, Tilson HA (1990) MK801 impedes the acquisition of spatial memory task in rats Pharmacol Biochem Behav 37:41-45.

McNaughton BL, Morris RG (1987) Hippocampal synaptic enhancement and information storage within a distributed memory system. Trends Neurosci 10:408-415.

McNaughton BL, Barnes CA, Meltzer J, Sutherland RJ (1989) Hippocampal granule cells are necessary for normal spatial learning but not for spatially-selective pyramidal cell discharge. Exp Brain Res 76:485-496.

Mizumori SJ, Ragozzino KE, Cooper BG, Leutgeb S (1999) Hippocampal representational organization and spatial context. Hippocampus 9:444-451.

Moser MB, Moser EI (1998) Distributed encoding and retrieval of spatial memory in the hippocampus. J Neurosci 18:7535-7542.

Moser M, Trommald M, Andersen P (1994) An increase in dendritic spine density on hippocampal CA1 pyramidal cells following spatial learning in adult rats suggest the formation of new synapses. Proc Natl Acad Sci USA 91:12673-12675.

Nadel L, Eichenbaum H (1999) Introduction to the special issue on place cells. Hippocampus 9:341-345.

Nerad L, Ramirez-Amaya V, Ormsby CE, Bermudez-Rattoni F (1996) Differential effects of anterior and posterior insular cortex lesions on the acquisition of conditioned taste aversion and spatial learning. Neurobiol Learn Mem 66:44-50.

Nyakas C, Luiten PG, Spencer DG, Traber J (1987) Detailed projection patterns of septal and diagonal band efferents to the hippocampus in the rat with emphasis on innervation of CA1 and dentate gyrus. Brain Res Bull 18:533-545.

Oliva Jr AA, Jiang M, Lam T, Smith KL, Swann JW (2000) Novel hippocampal interneuronal subtypes identified using transgenic mice that express green fluorescent protein in GABAergic interneurons. J Neurosci 20:3354-3368.

Paxinos G, Watson C (1986) The rat brain in stereotaxic coordinates. Orlando, FL: Academic.

Pikkarainen M, Ronkko S, Savander V, Insausti R, Pitkanen A (1999) Projections from the lateral, basal, and accessory basal nuclei of the amygdala to the hippocampal formation in rat. J Comp Neurol 403:229-260.

Poncer JC, McKinney RA, Gahwiler BH, Thompson SM (2000) Differential control of GABA release at synapses from distinct interneurons in rat hippocampus. J Physiol (Lond) 528:123-130.

Pleskacheva MG, Wolfer DP, Kupriyanova IF, Nikolenko DL, Scheffrahn H, Dell'Omo G, Lipp HP (2000) Hippocampal mossy fibers and swimming navigation learning in two vole species occupying different habitats. Hippocampus 10:17-30.

Ramírez-Amaya V, Escobar ML, Chao V, Bermúdez-Rattoni F (1999) 
Synaptogenesis of Mossy fibers induced by spatial water maze overtraining. Hippocampus 9:631-636.

Rapp PR, Stack EC, Gallagher M (1999) Morphometric studies of the aged hippocampus: I. Volumetric analysis in behavioral characterized rats. J Comp Neurol 403:459-470.

Rusakov DA, Davies HA, Harrison E, Diana G, Richter-Levin G, Bliss TVP, Stewart MG (1997) Ultrastructural synaptic correlates of spatial learning in rat hippocampus. Neuroscience 80:69-77.

Schwegler H, Mueller GG, Crusio WE, Szemes L, Seress L (1993) Hippocampal morphology and spatially related behavior in Long-Evans and CFY rats. Hippocampus 3:1-8.

Shors TJ, Miesegaes G, Beylin A, Zhao M, Rydel T, Gould E (2001) Neurogenesis in the adult is involved in the formation of trace memories. Nature 410:372-376.
Van-der-Zee CE, Rashid K, Le K, Moore KA, Stanisz J, Diamond J, Racine RJ, Fahnestock M (1995) Intraventricular administration of antibodies to nerve growth factor retards kindling and blocks mossy fiber sprouting in the adult rats. J Neurosci 15:5316-5323.

Woolf NJ (1998) A structural basis for memory storage in mammals. Prog Neurobiol 55:59-77.

Wuarin JP, Dudek FE (1996) Electrographic seizures and new recurrent excitatory circuits in the dentate gyrus of hippocampal slices from kainate-treated epileptic rats. J Neurosci 16:4436-4448.

Xavier GF, Oliveira-Filho FJB, Santos AMG (1999) Dentate gyrusselective colchicine lesion and disruption of performance in spatial tasks: difficulties in place strategy because of a lack of flexibility in the use of environmental cues? Hippocampus 9:668-681. 